\title{
Theoretical investigation of light transport through bidispersed magnetic colloid with field dependent relative refractive index
}

\author{
Keyur G. Khatsuriya \\ Shantilal Shah Engineering College, Bhavnagar, Gujarat-364060, India \\ Email: keyur.physics@gmail.com
}

Bidispersed magnetic colloid is composed of micrometer sized magnetic sphere dispersed in magnetic nanofluid. In this paper the variation in relative refractive index of bidispersed magnetic fluid was studied as a function of external magnetic field.

It is observed that the refractive index of monodispersed magnetic nanofluid $\left(n_{f}\right)$ increases with external magnetic field and saturated $[1,2]$. This is due to structure formation [3, 4]. This is not the case of bidispersed magnetic colloid ${ }^{5}$. Therefore; the modulation in refractive index for bidispersed ferrofluid can be explained on the basis of magnetically modulated dielectric constant $(\varepsilon)$. We have refractive index as $n=\sqrt{\varepsilon}$ where $\varepsilon=$ $\varepsilon_{0}+\varepsilon_{\infty} F(\alpha) ; \varepsilon_{0}$ and $\varepsilon_{\infty}$ are relative permittivity of the bidispersed ferrofluid at zero field and at infinite field (saturation value), $F(\alpha)=\alpha L^{2}(\alpha)$ $/ \alpha-L(\alpha), L(\alpha)=\operatorname{coth} \alpha-\alpha^{-1}, \alpha=\mu \mathrm{H} / k_{B} T, \mu$ is magnetic moment, $H$ is applied field, $k_{B}$ is Boltzmann constant and $T$ is absolute temperature. $F(\alpha)$ is derived using effective field [6]. $\mu=1$ at optical frequency. The relative refractive index $(n)$ is the ratio of refractive index of scatterer $\left(n_{s}\right)$ to the fluid $\left(n_{f}\right)$ [5]. Figure shows the variation in change of refractive indices with the external field.

Possible theoretical reasons are $(i)$ due to extinction of light in fluid as micro-sized magnetic particle will behave as Mie scatterer and nanomagnetic particle will behave as Rayleight scattere [5], (ii) due to optical limiting occurs for large difference in refractive indices of nano and micro sized magnetic particle, (iii) due to resonance in electric and magnetic field generated at the time of light transport. All above mentioned phenomenon may be possible during light transport results in extinction of light. Hence, with the increase in $n_{s}$ transmission of light decreases and $\Delta n$ saturates 'early'.

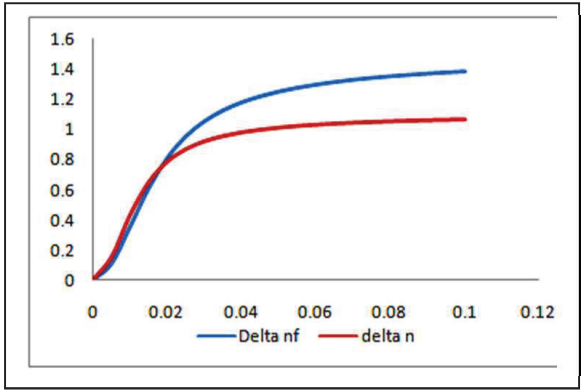

Figure 1: $\Delta n$ and $\Delta n_{f}$ for $n_{s}=3$

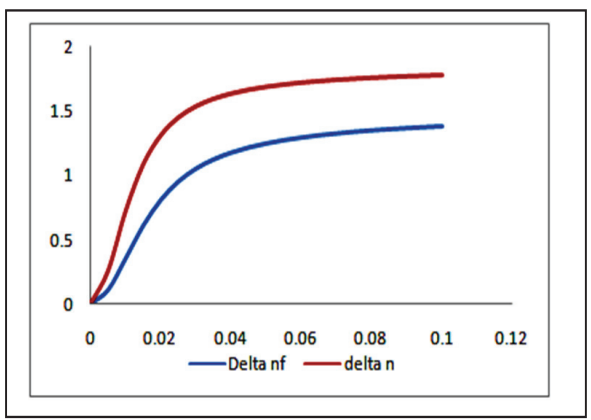

Figure 2: $\Delta n$ and $\Delta n_{f}$ for $n_{s}=5$

Figure 2 shows that as the refractive index $\left(n_{s}\right)$ of scatterer increases fewer magnetic fields required for saturation of $\Delta n$.

Present study elucidates the variation in refractive index with field at nano level. The study helps to design novel photonic devices.

\section{References}

1. S. Pu, X. Chen, Y. Chen, W. Liao, L. Chen and Y. Xia, Appl. Phys. Lett. 86, 171904(2005)

2. Y. F. Chen, S. Y. Yang, W. S. Tse, H. E. Horng, C. Y. Hong and H. C. Yang, Appl. Phys. Lett. 82, 3481(2003)

3. S. Y. Yang, J. J. Chieh, H. e. Horng, C. Y. Hong and H. C. Yang, Appl. Phys. Lett. 84, 5204(2004)

4. S. Taketomi, J. J. Appl. Phys. 22, 1137(1983)

5. Hem Bhatt, Rajesh Patel, Journal of Nanofluids, Vol.2, No. 3 (2013)

6. M. A. Martsenyuk, Yu. L. Raikher, M. I. Shliomis, Sov. Phys. JETP, 38, 413 (1974) 